\title{
UNIVERSITY OF LUND RADIOCARBON DATES XII
}

\author{
SÖREN HÅKANSSON
}

Radiocarbon Dating Laboratory, Department of Quaternary Geology, University of Lund, Sweden

\section{INTRODUCTION}

Most of the ${ }^{14} \mathrm{C}$ measurements reported here were made between October 1977 and October 1978. Equipment, measurement, and treatment of samples are as reported previously $(\mathrm{R}, 1968$, v 10, p 36-37; 1976, $\mathrm{v}$ 18, p 290) except for some minor improvements of the electronic equipment.

Age calculations are based on a contemporary value equal to $95 \%$ of the activity of NBS oxalic acid standard and on the conventional halflife for ${ }^{14} \mathrm{C}$ of 5568 yr. Results are reported in years before 1950 (years BP). Errors quoted $( \pm 1 \sigma)$ include standard deviations of count rates for the unknown sample, contemporary standard, and background. When measured activity is less than $2 \sigma$ above background, minimum age is given. Basis for calculation of age limit is measured net activity plus $3 \sigma$. If net activity is negative, only $+3 \sigma$ is used for age limit.

Corrections for deviations from $\delta^{13} \mathrm{C}=-25.0 \%$ in the PDB scale are applied for all samples; also for marine shells. The apparent age for marine material must be subtracted from our dates on such samples.

The remark "undersized; diluted", in Comments means the sample did not produce enough $\mathrm{CO}_{2}$ to fill the counter to normal pressure and "dead" $\mathrm{CO}_{2}$ from anthracite was introduced to make up the pressure. "\% sample" indicates amount of $\mathrm{CO}_{2}$ derived from the sample present in the diluted counting gas; the rest is "dead" $\mathrm{CO}_{2}$. Organic carbon content reported for bone samples is calculated from yield of $\mathrm{CO}_{2}$ by combustion of gelatine remaining after treatment. Organic carbon lost during treatment is not included in calculated percentage.

The description of each sample is based on information provided by the submitter.

\section{ACKNOWLEDGMENTS}

The author thanks Kerstin Lundahl for sample preparation and routine operation of the dating equipment, and $\mathrm{R}$ Ryhage and his staff at the mass-spectrometric laboratory of Karolinska Inst, Stockholm, for the ${ }^{13} \mathrm{C}$ analyses.

\section{SAMPLE DESCRIPTIONS}

I. GEOLOGIC SAMPLES

\section{Herrestads mosse series}

\section{A. Sweden}

Sediment and peat from bog Herrestads mosse, S Scania $\left(55^{\circ} 25.5^{\prime} \mathrm{N}\right.$, $13^{\circ} 52^{\prime}$ E). Coll 1975 and subm by J Mikaelsson, Dept Quaternary Geol, Univ Lund. Dated as part of study of water-level changes in area and vegetational development in surrounding region (Mikaelsson \& Liljegren, 
1978). Depths given refer to bog surface. All samples pretreated with HCl. Some received additional mild treatment with $\mathrm{NaOH}$.

Lu-1225. Herrestads mosse, $390 \mathrm{~cm}$

$$
9620 \pm 95
$$

$\delta^{13} \mathrm{C}=-26.7 \%$

Coarse detritus gyttja. Comment: additional mild $\mathrm{NaOH}$ treatment.

Lu-1226. Herrestads mosse, $380 \mathrm{~cm}$

$$
\begin{array}{r}
\mathbf{9 4 2 0} \pm \mathbf{9 5} \\
\delta^{13} C=-27.3 \% 0
\end{array}
$$

Coarse detritus gyttja.

Lu-1227. Herrestads mosse, $370 \mathrm{~cm}$

$$
9250 \pm 90
$$

Coarse detritus gyttja.

$$
\delta^{13} C=-26.1 \%
$$

Lu-1228. Herrestads mosse, $335 \mathrm{~cm}$

$$
8560 \pm 85
$$

$\delta^{13} C=-23.5 \%$

Coarse detritus gyttja. Comment: additional mild NaOH treatment.

Lu-1229. Herrestads mosse, $315 \mathrm{~cm}$

Drift gyttja.

$$
\begin{array}{r}
\mathbf{7 6 8 0} \pm \mathbf{8 0} \\
\delta^{13} C=-25.8 \% o
\end{array}
$$

\section{Lu-1230. Herrestads mosse, $305 \mathrm{~cm}$}

$7590 \pm 80$

Fine detritus gyttja with some root material.

Lu-1231. Herrestads mosse, $250 \mathrm{~cm}$

$$
\begin{array}{r}
6350 \pm 75 \\
\delta^{13} C=-26.5 \% 。
\end{array}
$$

Fine detritus gyttja. Comment: additional mild $\mathrm{NaOH}$ treatment.

\section{Lu-1232. Herrestads mosse, $200 \mathrm{~cm}$}

$$
\begin{array}{r}
\mathbf{6 0 9 0} \pm \mathbf{7 0} \\
\delta^{13} C=-26.8 \% o
\end{array}
$$

Fine detritus gyttja with few root hairs. Comment: additional mild $\mathrm{NaOH}$ treatment.

\section{Lu-1233. Herrestads mosse, $150 \mathrm{~cm}$}

$$
\begin{array}{r}
\mathbf{5 6 0 0} \pm \mathbf{7 0} \\
\delta^{1.3} C=-27.0 \% 0
\end{array}
$$

Fine detritus gyttja with some root material and few minute mollusk shells. Comment: additional mild $\mathrm{NaOH}$ treatment.

\section{Lu-1234. Herrestads mosse, $120 \mathrm{~cm}$}

$$
\begin{array}{r}
\mathbf{5 0 5 0} \pm \mathbf{6 5} \\
\delta^{13} C=-26.5 \% \text {. }
\end{array}
$$

Fine detritus gyttja with many minute mollusk shells. Comment: additional mild $\mathrm{NaOH}$ treatment. Carbonate from this sample was dated separately at $5940 \pm 125\left(\delta^{13} \mathrm{C}=-7.7 \%\right.$ o $)$.

\section{Lu-1235. Herrestads mosse, $45 \mathrm{~cm}$}

$$
\begin{array}{r}
3590 \pm \mathbf{6 0} \\
\delta^{13} C=-25.0 \% \text { o }
\end{array}
$$
ment.

Strongly humified peat. Comment: additional mild $\mathrm{NaOH}$ treat- 


\section{Olsäng series}

Peat and peaty drift gyttja from area with beach ridges (Mikaelsson, 1978) at Olsäng, SE Blekinge (56 $\left.12.5^{\prime} \mathrm{N}, 16^{\circ} 58.5^{\prime} \mathrm{E}\right)$. Coll 1976 and 1977 and subm by J Mikaelsson. Dated as part of study of chronology of beach ridges and of shoreline displacement in area. For stratigraphy of dated samples, see op cit, above, p 42-43, 47.

\section{Lu-1461. Olsäng I, Bp 27}

$$
\begin{array}{r}
5690 \pm 70 \\
\delta^{13} C=-26.4 \% \text { o }
\end{array}
$$

Strongly compacted peat from Boring Point 27. Comment: pretreated with $\mathrm{HCl}$ and $\mathrm{NaOH}$.

\section{Lu-1462. Olsäng II, Bp 23}

$$
\begin{array}{r}
9150 \pm 90 \\
\delta^{13} C=-26.6 \% \text { o }
\end{array}
$$

Highly humified Sphagnum peat from Boring Point 23. Comment: no pretreatment; small sample.

Lu-1463. Olsäng III, Bp 11

$$
\begin{array}{r}
9220 \pm 90 \\
\delta^{13} C=-26.8 \%
\end{array}
$$

Peat with some gyttja from Boring Point 11. Comment: no pretreatment; small sample.

\section{Lu-1460. Olsäng IV, Bp 13}

$$
\begin{array}{r}
9120 \pm 90 \\
\delta^{13} C=-26.0 \%
\end{array}
$$

Peaty drift gyttja from Boring Point 13. Comment: pretreated with $\mathrm{HCl}$ and $\mathrm{NaOH}$.

\section{Sämbosjön series (II)}

Sediment from Lake Sämbosjön, Halland, SW Sweden $\left(57^{\circ} 10^{\prime} \mathrm{N}\right.$, $\left.12^{\circ} 25^{\prime} \mathrm{E}\right)$. Coll 1976 and subm by G Digerfeldt, Dept Quaternary Geol, Univ Lund. Dated as complement to Sämbosjön series (R, 1978, v 20, p 418-419). Depths given are below sediment surface. Pretreated with HCl and $\mathrm{NaOH}$.

Lu-1280. Sämbosjön, 100 to $105 \mathrm{~cm}$

Detritus gyttja.

\section{Lu-1281. Sämbosjön, 60 to $65 \mathrm{~cm}$}

Detritus gyttja.

Lu-1418. Sämbosjön, 25 to $30 \mathrm{~cm}$

Detritus gyttja.

$$
\begin{array}{r}
\mathbf{1 5 0 0} \pm \mathbf{5 0} \\
\delta^{13} C=-28.2 \% \\
\mathbf{1 2 0 0} \pm \mathbf{5 0} \\
\delta^{13} C=-27.8 \% 0 \\
\mathbf{1 1 4 0} \pm \mathbf{5 0} \\
\delta^{13} C=-25.4 \% 0
\end{array}
$$

\section{Åsbotorpsjön series}

Sediment from Lake Åsbotorpsjön, Billingen, S Sweden $\left(58^{\circ} 25^{\prime} \mathrm{N}\right.$, $13^{\circ} 50^{\prime} \mathrm{E}$ ). Alt 280m; area 2.5ha; max depth 7.5m. Coll 1976 and subm by $G$ Digerfeldt. Dating is part of study of Late Weichselian develop- 
ment of lake and surrounding region. Samples come from profile in southern part of lake (Livingstone sampler, diam $10 \mathrm{~cm}$ ). Water depth ca $4 \mathrm{~m}$ at sampling point. Depths given are below sediment surface. All samples pretreated with HCl.

Lu-1448. Åsbotorpsjön, 587 to $589.5 \mathrm{~cm}$

$$
14,020 \pm 155
$$

Clay gyttja. Comment: sample undersized; diluted; $67 \%$ sample. (3 1-day counts.)

\section{Lu-1449. Åsbotorpsjön, 584 to $586 \mathrm{~cm}$}

$$
\begin{array}{r}
12,010 \pm 130 \\
\delta^{13} C=-25.0 \%
\end{array}
$$

Clay gyttja. Comment: sample undersized; diluted; $80 \%$ sample.

\section{Lu-1450. Åsbotorpsjön, 576 to $578 \mathrm{~cm}$}

$11,520 \pm 120$

$\delta^{13} C=-25.2 \%$

Clay gyttja. Comment: sample undersized; diluted; $88 \%$ sample.

\section{Lu-1451. Åsbotorpsjön, 569.5 to $571.5 \mathrm{~cm}$}

$10,440 \pm 105$

$\delta^{13} C=-23.9 \%$

Clayey gyttja. Comment: sample undersized; diluted; $92 \%$ sample.

Lu-1452. Åshotorpsjön, 566 to $568 \mathrm{~cm}$

Clayey gyttja.

Lu-1453. Åsbotorpsjön, 558 to $560 \mathrm{~cm}$

Clayey gyttja.

Lu-1454. Åsbotorpsjön, 548 to $550 \mathrm{~cm}$

Clayey gyttja.

\section{Lu-1455. Åsbotorpsjön, 538 to $540 \mathrm{~cm}$}

Detritus gyttja.

\section{Lu-1456. Åsbotorpsjön, 522 to $524 \mathrm{~cm}$}

Detritus gyttja.

\section{Lillsjön series}

Sediment from Lake Lillsjön, Stockholm (59 $\left.20^{\prime} \mathrm{N}, 17^{\circ} 57^{\prime} \mathrm{E}\right)$. Alt $0.3 \mathrm{~m}$; area 12ha; max depth $2.9 \mathrm{~m}$. Coll 1976 and subm by $\mathrm{G}$ Digerfeldt. Dating is part of study of isolation and recent development of lake. Samples come from profile in central part of lake (Livingstone corer, diam $6 \mathrm{~cm}$ ). Water depth $2.9 \mathrm{~m}$ at sampling point. Depths given are below sediment surface. Pretreated with HCl. All samples undersized; diluted. Amount of $\mathrm{CO}_{2}$ from sample is given in Comments below as "\% sample". 
Lu-1430. Lillsjön, 90 to $92.5 \mathrm{~cm}$

Clay gyttja. Comment: $75 \%$ sample.

Lu-1429. Lillsjön, 70 to $72.5 \mathrm{~cm}$

Clay gyttja. Comment: $86 \%$ sample.

Lu-1428. Lillsjön, 50 to $52.5 \mathrm{~cm}$

Clay gyttja. Comment: $91 \%$ sample. $\mathbf{8 5 0} \pm \mathbf{5 5}$

$\delta^{13} \mathrm{C}=-28.3 \%$

$$
\mathbf{7 8 0} \pm \mathbf{5 0}
$$$$
\delta^{13} C=-29.3 \%
$$

$$
\mathbf{6 4 0} \pm \mathbf{5 0}
$$$$
\delta^{13} \mathrm{C}=-28.7 \%
$$

$$
\mathbf{3 2 0} \pm \mathbf{5 0}
$$

$\delta^{13} \mathrm{C}=-18.7 \%$ o

Algae gyttja from 95 to $97.5 \mathrm{~cm}$ below sediment surface in profile in central part of Lake Laduviken, Stockholm $\left(59^{\circ} 22^{\prime} \mathrm{N}, 18^{\circ} 05^{\prime} \mathrm{E}\right)$. Alt $0.7 \mathrm{~m}$; area 6ha; max depth $1.5 \mathrm{~m}$. Coll 1976 and subm by G Digerfeldt. Dated as part of study of recent development of lake. Water depth $1.5 \mathrm{~m}$ at sampling point. Pretreated with HCl. Comment: $95 \%$ sample.

\section{Lu-1427. Långsjön}

$490 \pm 50$

$$
\delta^{13} \mathrm{C}=-30.1 \%
$$

Detritus gyttja from 52.5 to $55 \mathrm{~cm}$ below sediment surface in profile in deepest part of Lake Långsjön, Stockholm $\left(59^{\circ} 16^{\prime} \mathrm{N}, 17^{\circ} 58^{\prime} \mathrm{E}\right)$. Coll 1976 and subm by G Digerfeldt. Dated as complement to Långsjön series (R, 1978, v 20, p 420-421). Pretreated with HCl.

\section{Sjömyretjärn series}

Sediment from Lake Sjömyretjärn, Dalsland, W Sweden $\left(58^{\circ} 48^{\prime} \mathrm{N}\right.$, $\left.12^{\circ} 05^{\prime} \mathrm{E}\right)$. Alt 152m; area 10ha; max depth $4.55 \mathrm{~m}$. Coll 1977 and subm by $\mathrm{G}$ Digerfeldt. Dating is part of study of Holocene development of lake and vegetational history of surrounding region. Samples come from profile in central part of lake (Livingstone corer, diam $10 \mathrm{~cm}$ ). Water depth $4.55 \mathrm{~m}$ at sampling point. Depths given are below sediment surface. All samples pretreated with HCl. Lu-1418, -1420, -1491, and -1492 received additional treatment with $\mathrm{NaOH}$.

Lu-1486. Sjömyretjärn, 405 to $410 \mathrm{~cm}$

Clay gyttja.

Lu-1487. Sjömyretjärn, 375 to $380 \mathrm{~cm}$

Clayey gyttja.

Lu-1488. Sjömyretjärn, 345 to $350 \mathrm{~cm}$

Clayey gyttja.

Lu-1489. Sjömyretjärn, 315 to $320 \mathrm{~cm}$

Detritus gyttja.

$$
\begin{array}{r}
\mathbf{9 7 5 0} \pm \mathbf{9 0} \\
\delta^{13} C=-23.5 \%
\end{array}
$$

$$
\begin{array}{r}
9500 \pm \mathbf{9 0} \\
\delta^{13} C=-22.5 \%
\end{array}
$$

$$
\begin{array}{r}
9630 \pm 90 \\
\delta^{13} C=-23.1 \%
\end{array}
$$

$$
\begin{array}{r}
\mathbf{8 8 8 0} \pm \mathbf{8 5} \\
\delta^{13} C=-24.0 \%
\end{array}
$$


Lu-1490. Sjömyretjärn, 275 to $280 \mathrm{~cm}$

$7680 \pm 75$

Detritus gyttja.

Lu-1491. Sjömyretjärn, 235 to $240 \mathrm{~cm}$

$7050 \pm 90$

$\delta^{13} \mathrm{C}=-29.7 \%$

Detritus gyttja. Insoluble fraction. Comment: undersized; diluted; $72 \%$ sample.

Lu-1491A. Sjömyretjärn, 235 to $240 \mathrm{~cm}$, soluble $7030 \pm 75$

Acid-precipitated part of $\mathrm{NaOH}$-soluble fraction.

$\delta^{13} \mathrm{C}=-29.3 \%$

Lu-1492. Sjömyretjärn, 195 to $200 \mathrm{~cm}$

Detritus gyttja.

$\mathbf{5 4 9 0} \pm \mathbf{6 5}$
$\delta^{13} C=-29.9 \%$

Lu-1493. Sjömyretjärn, 155 to $160 \mathrm{~cm}$

Detritus gyttja.

Lu-1494. Sjömyretjärn, 115 to $120 \mathrm{~cm}$

Detritus gyttja.

Lu-1420. Sjömyretjärn, 75 to $80 \mathrm{~cm}$

Detritus gyttja.

Lu-1419. Sjömyretjärn, 35 to $40 \mathrm{~cm}$

Detritus gyttja.

\section{Central Blekinge series ( $I$ )}

Sediment from lakes in Central Blekinge, SE Sweden. All samples except Lu-1554 coll 1977 and subm by S Björck, Dept Quaternary Geol, Univ Lund. Dating is part of study of age of isolation level in lakes of different alt and of pollen zonation in area. Samples are from cores taken with Livingstone sampler, diam $10 \mathrm{~cm}$, except Lu-1554 (diam $6 \mathrm{~cm}$ ). Depths refer to water surface, except for overgrown Lake Paddegölen, where depths refer to quagmire surface. Most of the samples were undersized and were diluted. Amount of $\mathrm{CO}_{2}$ from sample is given in Comments below as "\% sample". All samples pretreated with HCl.

\section{Galtsjön}

$\left(56^{\circ} 13^{\prime} \mathrm{N}, 15^{\circ} 13^{\prime} \mathrm{E}\right)$, alt $32 \mathrm{~m}$. Water depth ca $5 \mathrm{~m}$ at sampling point.
Lu-1422. Galtsjön, 794 to $797 \mathrm{~cm}$
$11,000 \pm 175$

Muddy clay, overlying isolation level. Comment: $48 \%$ sample. 
Lu-1423. Galtsjön, 778.5 to $781.5 \mathrm{~cm}$

Lu-1424. Galtsjön, 762 to $765 \mathrm{~cm}$

Clay gyttja. Comment: $91 \%$ sample.

Lu-1425. Galtsjön, 759 to $761 \mathrm{~cm}$

Clayey gyttja. Comment: $67 \%$ sample.

\section{Paddegölen}

$\left(56^{\circ} 11^{\prime} \mathrm{N}, 15^{\circ} 13^{\prime} \mathrm{E}\right)$, alt $27 \mathrm{~m}$.

Lu-1471. Paddegölen, 615 to $620 \mathrm{~cm}$

Clay. Comment: $24 \%$ sample. (4 1-day counts.)

Lu-1472. Paddegölen, 596 to $600 \mathrm{~cm}$
Muddy clay. Comment: $83 \%$ sample.

$10,470 \pm 110$

$\delta^{13} C=-22.3 \%$

$10,050 \pm 100$

$\delta^{18} C=-22.0 \%$ o

$10,020 \pm 120$

$\delta^{13} C=-22.8 \%$ o

$13,670 \pm 295$

$\delta^{13} C=-21.9 \%$

$11,310 \pm 175$

$\delta^{13} \mathrm{C}=-22.5 \%$ counts.)

Clay, underlying isolation level. Comment: $38 \%$ sample. (3 1-day

Lu-1473. Paddegölen, 592 to $595 \mathrm{~cm}$

$10,820 \pm 110$ $\delta^{13} \mathrm{C}=-19.7 \%$

Clay gyttja, at isolation level. Comment: $84 \%$ sample.

Lu-1474. Paddegölen, 576 to $578.5 \mathrm{~cm}$

$10,100 \pm 95$ $\delta^{13} C=-21.0 \%$

Fine detritus gyttja.

\section{Kroksjön}

$\left(56^{\circ} 16^{\prime} \mathrm{N}, 15^{\circ} 01^{\prime} \mathrm{E}\right)$, alt $46 \mathrm{~m}$. Water depth ca $2 \mathrm{~m}$ at sampling point.

Lu-1477. Kroksjön, 510 to $515 \mathrm{~cm}$

$$
13,920 \pm 340
$$
$\delta^{13} \mathrm{C}=-20.7 \%$ counts.)

Clay, overlying isolation level. Comment: $26 \%$ sample. (3 1-day

Lu-1479. Kroksjön, 491 to $494 \mathrm{~cm}$

$$
11,710 \pm 115
$$$$
\delta^{13} C=-21.2 \%
$$

Clay gyttja. Comment: $67 \%$ sample. (3 1-day counts.)

Lu-1480. Kroksjön, 484 to $487 \mathrm{~cm}$

$11,100 \pm 130$ $\delta^{13} C=-22.6 \%$

Clay gyttja. Comment: $68 \%$ sample.

Lu-1481. Kroksjön, 468 to $472 \mathrm{~cm}$

$10,460 \pm 95$

Clay gyttja. 
Lu-1482. Kroksjön, 456 to $459 \mathrm{~cm}$

$10,330 \pm 95$

Clay gyttja.

\section{Bredsjön} point.

$\left(56^{\circ} 10^{\prime} \mathrm{N}, 15^{\circ} 12^{\prime} \mathrm{E}\right)$, alt $12 \mathrm{~m}$. Water depth ca $1.7 \mathrm{~m}$ at sampling

Lu-1555. Bredsjön, 533 to $540 \mathrm{~cm}$

$14,310 \pm 265$

$\delta^{13} \mathrm{C}=-23.7 \%$ sample.

Slightly muddy clay, underlying isolation level. Comment: $43 \%$

Lu-1556. Bredsjön, 526 to $530 \mathrm{~cm}$

$11,380 \pm 160$

$\delta^{13} \mathrm{C}=-22.8 \%$

Slightly muddy clay, at isolation level. Comment: $44 \%$ sample. (3 1-day counts.)

\section{Lu-1557. Bredsjön, 522 to $525 \mathrm{~cm}$}

$10,230 \pm 105$

$\delta^{13} \mathrm{C}=-25.3 \%$

Clay gyttja, overlying isolation level. Comment: $86 \%$ sample.

\section{Logylet} point.

$\left(56^{\circ} 18^{\prime} \mathrm{N}, 14^{\circ} 59^{\prime} \mathrm{E}\right)$, alt $61 \mathrm{~m}$. Water depth ca $5.5 \mathrm{~m}$ at sampling

Lu-1444. Logylet II, 970 to $974 \mathrm{~cm}$

$11,430 \pm 140$

Clay gyttja. Comment: $69 \%$ sample.

$$
\delta^{13} \mathrm{C}=-23.6 \%
$$

\section{Sjalbredan}

$\left(56^{\circ} 17^{\prime} 30^{\prime \prime} \mathrm{N}, 15^{\circ} 01^{\prime} 30^{\prime \prime} \mathrm{E}\right)$, alt $42 \mathrm{~m}$. Coll 1978 by R Liljegren and J Mikaelsson; subm by S Björck.

Lu-1554. Sjalbredan, 577 to $581 \mathrm{~cm} \quad 10,990 \pm 135$

$\delta^{13} \mathrm{C}=-21.1 \%$

Clay gyttja, overlying isolation level. Comment: $53 \%$ sample. (3 1-day counts).

\section{Öppenskär I series}

Peat from soil profile in Calluna heath on Öppenskär I., Torhamn archipelago, SE Blekinge (56 $\left.04^{\prime} \mathrm{N}, 15^{\circ} 47^{\prime} \mathrm{E}\right)$. Coll 1977 and subm by B E Berglund, Dept Quaternary Geol, Univ Lund. Area described by Berglund (1966, p 107-108). Pollen diagram from profile discussed by Berglund (1978). Depths refer to soil surface. Pretreated with $\mathrm{HCl}$ and $\mathrm{NaOH}$.

Lu-1485. Öppenskär I: 1,25 to $26.5 \mathrm{~cm}$, insoluble $130 \pm 45$ $\delta^{13} C=-26.0 \%$

Insoluble fraction of fen peat from just above mineral soil and below Calluna peat. 
Lu-1485A. Öppenskär I:1, 25 to $26.5 \mathrm{~cm}$, soluble $220 \pm 45$

$\delta^{13} C=-26.6 \%$

Acid-precipitated part of NaOH-soluble fraction of same peat. Comment (BEB): dates expansion of Calluna and possibly a local embogging. $\mathrm{Lu}-1485 \mathrm{~A}$ indicates that younger roots may influence date of insoluble fraction.

\section{Vieskär III series}

Charcoal and peat from fen profile in bedrock depression on Vieskär I., Torhamn archipelago, SE Blekinge (56 $\left.04^{\prime} \mathrm{N}, 15^{\circ} 47^{\prime} \mathrm{E}\right)$. Coll 1977 and subm by B E Berglund. Pollen diagram discussed by Berglund (1978). Depths refer to fen surface. Peat pretreated with $\mathrm{HCl}$ and $\mathrm{NaOH}$.

Lu-1468. Vieskär III:1, 34cm

$$
\begin{array}{r}
1210 \pm 50 \\
\delta^{13} C=-26.5 \% \text { o }
\end{array}
$$

Charcoal of Betula and Salix or Populus, id by $\mathrm{T}$ Bartholin, from drift gyttja layer. Dates clearing phase, expansion of Calluna heath, and local embogging. Comment: no pretreatment; small sample; diluted; $65 \%$ sample. (3 1-day counts.)

Lu-1484. Vieskär III:2, 27 to $28.5 \mathrm{~cm}$, insoluble $420 \pm 45$ $\delta^{13} \mathrm{C}=-27.7 \%$

Insoluble fraction of fen peat which dates end of Calluna phase and expansion of grass-juniper heath.

Lu-1484A. Vieskär III:2, 27 to $28.5 \mathrm{~cm}$, soluble $420 \pm 45$

Acid-precipitated part of $\mathrm{NaOH}$-soluble fraction of same peat.

\section{Lummelunda Cave series}

Gyttja from Lummelunda Cave, N Gotland (57 $44^{\prime} 18^{\prime \prime}$ N, $18^{\circ} 24^{\prime}$ 44" E). Coll 1977 by R Engh; subm by L Engh, Dept Phys Geog, Univ Lund. Dated as part of study of chronology of cave deposits. In samples were some small fresh-water mollusks. Pretreated with HCl.

\section{Lu-1511. Lummelunda Cave, Sample 8}

$$
\begin{array}{r}
\mathbf{3 5 7 0} \pm \mathbf{4 5} \\
\delta^{13} C=-24.1 \% \text { \% }
\end{array}
$$

Sample from $7 \mathrm{~cm}$ thick gyttja layer in Mailbox Hall, overlain by silt and underlain by travertine alternating with silt. Comment: 3 1-day counts.

\section{Lu-1512. Lummelunda Cave, Sample 24}

$$
\begin{array}{r}
\mathbf{3 5 3 0} \pm \mathbf{6 0} \\
\delta^{13} C=-24.3 \% \text { }
\end{array}
$$

Sample from $22 \mathrm{~cm}$ thick gyttja layer in Siphon Hall, overlain by coarse sand and underlain by ca $1 \mathrm{~m}$ laminated sandy sediments. 
Lu-1513. Strimasund

$\mathbf{8 0 8 0} \pm \mathbf{8 0}$

$\delta^{13} \mathrm{C}=-26.9 \%$

Peat from rd cutting $700 \mathrm{~m} \mathrm{E}$ of Strimasund farm at Rd E79, SW Lappland $\left(66^{\circ} 03^{\prime} 10^{\prime \prime} \mathrm{N}, 14^{\circ} 52^{\prime} 09^{\prime \prime} \mathrm{E}\right)$. Coll 1976 and subm by L Engh. Peat is underlain by glaciofluvial deposits in fossil canyon.

\section{Svedaskogen series}

Barnacle and bivalve shells from Svedaskogen, ca $3 \mathrm{~km} \mathrm{~N}$ of Fjärås church, Halland (57 $\left.28^{\prime} 45^{\prime \prime} \mathrm{N}, 12^{\circ} 10^{\prime} 30^{\prime \prime} \mathrm{E}\right)$. Coll 1977 and subm by A Hillefors, Dept Phys Geog, Univ Lund. Dated as part of study of deglaciation of area (Hillefors, 1975; R, 1976, v 18, p 295-297).

\section{Lu-1446. Svedaskogen, Sample $1: 1977$}

$13,290 \pm 125$ $\delta^{13} C=+0.1 \%$ o

Shells (Balanus sp) from silty clay underlain by glaciofluvial material and overlain by wave-washed sand and gravel. Some specimens still had all side plates attached to basal plate. Sample also contained few shells of bivalves (Macoma calcarea, Hiatella arctica, Mya truncata, and Mytilus edulis); not used for dating. Comment: outer $45 \%$ removed by acid leaching.

\section{Lu-1447. Svedaskogen, Sample 2:1977}

$12,970 \pm 120$

$\delta^{18} \mathrm{C}=-0.2 \%$

Barnacle shells (Balanus spp) and few fragments of bivalve shells (Mytilus sp) from glacial-tectonized glacial-marine clay overlain by till. Comment: outer $13 \%$ removed by acid leaching. Sample undersized; diluted; $78 \%$ sample. (3 1-day counts.)

General Comment: corrections for deviations from $\delta^{13} \mathrm{C}=-25 \%$ PDB are applied also for shell samples. No corrections are made for apparent age of shells of living marine organisms. For apparent age of recent mollusk shells, see R, 1975, v 17, p 183-184 and Håkansson (1975b).

\section{Iso-Mustajärvi series}

\section{B. Finland}

Sediment from Lake Iso-Mustajärvi, Ylitornio, Finland (66 $66^{\circ} 13^{\prime} 30^{\prime \prime}$ N, $23^{\circ} 48^{\prime} \mathrm{E}$ ). Alt ca $75 \mathrm{~m}$. Coll 1976 and subm by M Hjelmroos, Dept Quaternary Geol, Univ Lund. Dated as complement to Merijänjärvi and Pilpajärvi series (R, 1978, v 20, p 427-428). Samples are from core taken with Livingstone sampler. Depths given are below sediment surface. Water depth ca $1.3 \mathrm{~m}$ at sampling point. Pretreated with $\mathrm{HCl}$.

\section{Lu-1431. Iso-Mustajärvi I, 206 to $215 \mathrm{~cm}$}

$$
\begin{array}{r}
\mathbf{5 3 8 0} \pm \mathbf{6 5} \\
\delta^{13} C=-28.8 \% \text { \% }
\end{array}
$$

Slightly clayey gyttja. First impact of human influence; max of Plantago major, Urtica, and Humulus. 
Lu-1432. Iso-Mustajärvi II, 84 to $93 \mathrm{~cm}$

$$
\begin{array}{r}
\mathbf{3 9 1 0} \pm \mathbf{6 0} \\
\delta^{1.3} \mathrm{C}=-29.1 \% \text {. }
\end{array}
$$
limit.

Gyttja. Grazing stage with Plantago lanceolata; empiric Calluna

\section{Lu-1421. Haugalia, Kvam \\ C. Norway}

$$
\begin{array}{r}
>\mathbf{4 3 , 4 0 0} \\
\delta^{13} \mathrm{C}=-19.5 \%
\end{array}
$$

Collagen from mammoth tusk fragment from Skarsanden gravel pit at Haugalia, Kvam, Gudbrandsdalen (61 $\left.39^{\prime} 36^{\prime \prime} \mathrm{N}, 9^{\circ} 37^{\prime} 57^{\prime \prime} \mathrm{E}\right)$. Coll June 1977 and subm by K Garnes, Geol Dept, Univ Bergen, Norway. Deposits at Haugalia studied by submitter (Bergersen \& Garnes, 1971). For other mammoth dates from area, see Berglund et al (1976, p 185) and Garnes (1978, p 197). Collagen extracted as described previously (R, 1976, v 18, p 290) including $\mathrm{NaOH}$ treatment. Organic carbon content: $5.8 \%$. Comments: 4 1-day counts. Three $\sigma$ were used for calculation of min age. Average net activity was almost exactly zero. (KG): in Garnes (1978, $\mathrm{p}$ 197) because of printing error this date is quoted as a definite date 43,400 вр.

\section{Sotra series (II)}

Sediment from small lakes on Sotra I., Hordaland, W Norway. Coll 1977 by K Krzywinski, B Stabell, M Kvamne, and E Risnes; subm by B Stabell and K Krzywinski, Bot Mus, Univ Bergen. Dated as complement to Sotra series (R, 1978, v 20, p 423-424). Preliminary repts of study pub by Krzywinski and Stabell (1978) and Berge et al (1978). Acid-precipitated part of $\mathrm{NaOH}$-soluble fraction used for dating, except for Lu-1552 (dated on total organic material).

Lu-1495A. Sotra No. 10526/27

$$
\begin{array}{r}
\mathbf{2 7 9 0} \pm \mathbf{5 5} \\
\delta^{13} \mathrm{C}=-27.6 \%
\end{array}
$$

Lacustrine gyttja from Bakketj $\phi \mathrm{nn}\left(60^{\circ} 23^{\prime} \mathrm{N}, 4^{\circ} 59^{\prime} \mathrm{E}\right)$ overlying isolation contact.

Lu-1496A. Sotra No. 10613

$$
\begin{array}{r}
4880 \pm \mathbf{6 5} \\
\delta^{13} C=-28.2 \% 0
\end{array}
$$

Lacustrine gyttja from "Einerhaugen”, Tjørna $\left(60^{\circ} 21^{\prime} \mathrm{N}, 4^{\circ} 59^{\prime} \mathrm{E}\right)$, overlying isolation contact formed after Tapes-transgression.

Lu-1497A. Sotra No. 10648/49

Lacustrine gyttja from "Einerhaugen", Tjфrna, underlying ingression contact.

Lu-1498A. Sotra No. 10665

$$
\begin{array}{r}
9460 \pm 90 \\
\delta^{13} C=-22.4 \% o
\end{array}
$$

Lacustrine gyttja from "Einerhaugen", Tjørna, overlying isolation contact formed during lst postglacial regression. 
Lu-1527A. Sotra No. 10442

$\mathbf{2 2 4 0} \pm \mathbf{5 5}$

$\delta^{1.3} \mathrm{C}=-18.3 \%$

Lacustrine gyttja from Angeltveitvatnet, Angeltveit $\left(60^{\circ} 24^{\prime} \mathrm{N}, 5^{\circ} 00^{\prime}\right.$ E), overlying isolation contact. Comment: sample undersized; diluted; $81 \%$ sample.

Lu-1528A. Sotra No. 10730

$$
\mathbf{2 4 9 0} \pm \mathbf{5 5}
$$

$\delta^{13} C=-29.2 \%$

Lacustrine gyttja from Skrubbisvatn, Telavåg $\left(60^{\circ} 15^{\prime} \mathrm{N}, 4^{\circ} 59^{\prime} \mathrm{E}\right)$, overlying isolation contact.

\section{Lu-1529A. Sotra No. $12006 \quad 10,260 \pm 100$ \\ $\delta^{13} \mathrm{C}=-23.6 \%$}

Lacustrine gyttja from Tresskj $\phi \mathrm{nn}$, Kårtveit $\left(60^{\circ} 23^{\prime} \mathrm{N}, 5^{\circ} 00^{\prime} \mathrm{E}\right)$, overlying isolation contact. Comment: sample undersized; diluted; $87 \%$ sample.

Lu-1530A. Sotra No. 12168

$$
\begin{array}{r}
9920 \pm 90 \\
\delta^{13} C=-23.5 \%
\end{array}
$$

Lacustrine gyttja from Sekkingstadtj $\phi n n$, Sekkingstad $\left(60^{\circ} 21^{\prime} \mathrm{N}, 5^{\circ}\right.$ $00^{\prime} \mathrm{E}$ ), overlying isolation contact.

Lu-1552. Sotra No. 10904

$$
\begin{array}{r}
\mathbf{1 0 , 8 7 0} \pm \mathbf{1 9 5} \\
\delta^{13} \mathrm{C}=-23.9 \%
\end{array}
$$

Sediment from ingression contact in Kvemavatn, Fjaereide $\left(60^{\circ} 22^{\prime}\right.$ $\left.\mathrm{N}, 5^{\circ} 04^{\prime} \mathrm{E}\right)$. Comment: very small organic content, hence dated on total organic material. Insoluble fraction combusted at $<650^{\circ} \mathrm{C}$. Sample diluted; $29 \%$ sample. (3 1-day counts.)

Lu-1553A. Sotra No. 12237

$$
\begin{array}{r}
\mathbf{6 0 5 0} \pm \mathbf{7 0} \\
\delta^{1.3} C=-28.4 \%
\end{array}
$$

Sediment from Torkevikstj $\phi \mathrm{nn}\left(60^{\circ} 23^{\prime} \mathrm{N}, 4^{\circ} 58^{\prime} \mathrm{E}\right)$; mainly lacustrine gyttja but with a thin layer showing marine influence and representing absolute max of Tapes-transgression.

\section{Lu-1433. Flateyjardalur}

\section{Iceland}

Gyttja from lake at Flateyjardalur, N Iceland $\left(66^{\circ} 05^{\prime} 55^{\prime} \mathrm{N}, 17^{\circ} 54^{\prime}\right.$ $20^{\prime \prime} \mathrm{W}$ ). Coll 1977 by $\mathrm{H}$ Norddahl and G Hjaltason; subm by $\mathrm{H}$ Norddahl, Dept Quaternary Geol, Univ Lund. Dated as part of study of deglaciation of area. Comment: no pretreatment; small sample; diluted; $51 \%$ sample. (3 1-day counts.) 
Lu-1506. Sengstackes Bugt

\section{$\mathbf{4 4 , 7 0 0}$}

Large shells (Mya truncata) from clay reaching ca $+30 \mathrm{~m}$ SW of Sengstackes Bugt, N Shannon Ö, NE Greenland ( $\left.75^{\circ} 20^{\prime} \mathrm{N}, 18^{\circ} 26^{\prime} \mathrm{W}\right)$. Coll 1976 by C Hjort and H Bruch; subm by C Hjort, Dept Quaternary Geol, Univ Lund. Dated as complement to East Greenland Series I through VI (R, 1972, v 14, p 388-390; 1973, v 15, p 504-507; 1974, v 16, p 319-322; 1975, v 17, p 184-187; 1976, v 18, p 301-303; 1978; v 20, p 424427). Comment: outer $58 \%$ removed by acid leaching. (3 1-day counts.)

\section{Lu-1508. Kaffiöyra}

F. Spitsbergen

$$
\begin{array}{r}
9700 \pm 90 \\
\delta^{13} C=-16.5 \%
\end{array}
$$

Collagen from well-preserved unid. whale bone from surface of elev marine terrace, alt +6 to $7 \mathrm{~m}$, ca $700 \mathrm{~m}$ from recent sea cliff at Kaffiöyra, Spitsbergen $\left(78^{\circ} 36^{\prime} \mathrm{N}, 12^{\circ} 10^{\prime} \mathrm{E}\right)$. Coll 1975 by E Drozdowski and J Szypryczyński; subm by J Szupryczyński, Inst Geog, Polish Acad Sci, Toruń, Poland. Dated as part of continued study of glacial history of Spitsbergen (Klimaszewski, 1960). Comment: organic carbon content: $7.5 \%$.

\section{Lu-1507. Male Slońca}

\section{G. Poland}

$$
\begin{aligned}
&>\mathbf{3 9 , 5 0 0} \\
& \delta^{13} C=-1.4 \%
\end{aligned}
$$

Thick shell fragments, mostly of Arctica (Cyprina) islandica, from glaciofluvial deposits at Małe Słońca, $S$ of Tczew in lower Vistula valley, N Poland (54 $\left.03^{\circ} \mathrm{N}, 18^{\circ} 50^{\prime} \mathrm{E}\right)$. Coll 1977 and subm by E Drozdowski, Inst Geog, Polish Acad Sci, Toruń, Poland. Dated as complement to Lower Vistula valley series (R, 1976, v 18, p 303-304; 1978, v 20, p 429). Comment: outer $67 \%$ removed by acid leaching. (4 1-day counts.)

\section{Lu-1483. Genève}

$$
\text { H. Switzerland }
$$

$$
\begin{array}{r}
>\mathbf{4 4 , 5 0 0} \\
\delta^{13} \mathrm{C}=-24.9 \% 0
\end{array}
$$

Lignite from sec exposed at Pont de Sous-Terre (Jayet \& Amberger, 1969, p 630-632) in town of Geneva $\left(46^{\circ} 12^{\prime} 16^{\prime \prime} \mathrm{N}, 6^{\circ} 07^{\prime} 56^{\prime \prime} \mathrm{E}\right)$. Coll 1968 and subm by G F Amberger, Service Cantonal de Geol, Geneva, Switzerland. Stratigraphy, fauna, and flora described by Jayet and submitter (op cit, above); pollen study by Girard (1970). Comment: sample pretreated with $\mathrm{HCl}$ and $\mathrm{NaOH}$. (5 1-day counts).

\section{Varris series}

II. ARGHAEOLOGIC SAMPLES

\section{Sweden}

Charcoal from Sites Raä 518b and Raä 503 at Varris, Lake Malgo-

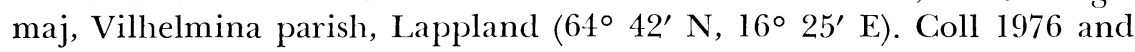


subm by L G Spång, Västerbottens Mus, Umeå. Dated as part of archaeol study of Stone age sites at Lake Malgomaj. Lu-1382 received normal pretreatment with $\mathrm{HCl}$ and $\mathrm{NaOH}$; all other samples were small and received only mild pretreatment with $\mathrm{NaOH}$ and HCl.

Lu-1374. Varris, Raä 518b, K 1 $950 \pm 50$ $\delta^{13} \mathrm{C}=-23.8 \%$

Charcoal from earth oven in piled up sand bank; depth ca $15 \mathrm{~cm}$.

\section{Lu-1375. Varris, Raä 518b, K 2}

$940 \pm 55$

$\delta^{13} \mathrm{C}=-24.7 \%$

Charcoal from same earth oven as Lu-1374; depth ca $25 \mathrm{~cm}$. Comment: sample undersized; diluted; $80 \%$ sample.

Lu-1376. Varris, Raä 518b, K 3

$$
\begin{array}{r}
1160 \pm 55 \\
\delta^{13} C=-24.2 \%
\end{array}
$$

Charcoal from same earth oven as Lu-1374; depth ca $40 \mathrm{~cm}$. Comment: sample undersized; diluted; $81 \%$ sample.

\section{Lu-1377. Varris, Raä 518b, K 4}

$$
\begin{array}{r}
1090 \pm 50 \\
\delta^{13} C=-24.9 \%
\end{array}
$$

Charcoal from same earth oven as Lu-1374; depth ca $45 \mathrm{~cm}$.

\section{Lu-1383. Varris, Raä 503, K 2}

$$
\begin{array}{r}
\mathbf{1 5 7 0} \pm \mathbf{5 0} \\
\delta^{13} \mathrm{C}=-24.8 \%
\end{array}
$$

Charcoal from bottom of earth oven with heat-cracked stone.

\section{Vojmsjöluspen series}

Charcoal from Site Raä 554 at Vojmsjöluspen, Vilhelmina parish, Lappland $\left(64^{\circ} 52^{\prime} \mathrm{N}, 16^{\circ} 45^{\prime} \mathrm{E}\right)$. Coll 1976 by L G and K Spång; subm by L G Spång. Dated as part of archaeol study of Stone age sites at Lake Vojmsjön. All samples were small and received therefore only mild pretreatment with $\mathrm{NaOH}$ and HCl. All except Lu-1381 undersized; diluted. Amount of $\mathrm{CO}_{2}$ from sample is given in Comments below as "\% sample".

\section{Lu-1373. Vojmsjöluspen, Raä 554, Sample $1 \quad 5540 \pm 75$

$$
\delta^{13} \mathrm{C}=-24.5 \%
$$

Charcoal from layer with soot and heat-cracked stone ca $30 \mathrm{~cm}$ below surface. Sq X108, Y322; +416.53 to 416.68. Comment: $86 \%$ sample.

\section{Lu-1378. Vojmsjöluspen, Raä 554, Sample 2}

$$
\begin{array}{r}
\mathbf{5 3 2 0} \pm \mathbf{7 0} \\
\delta^{13} \mathrm{C}=-23.7 \%
\end{array}
$$

Charcoal from refuse pit in till, depth ca $50 \mathrm{~cm}$. Sq X110, Y316; +416.17, K 1. Assoc with quartzite scrapers. Comment: $93 \%$ sample.

Lu-1379. Vojmsjöluspen, Raä 554, Sample 3

$$
\begin{array}{r}
5150 \pm 65 \\
\delta^{13} C=-23.6 \%
\end{array}
$$

Charcoal from bank of heat-cracked stone ca $50 \mathrm{~cm}$ below surface. Sq X107, Y321; +416.48. Comment: $60 \%$ sample. (4 1-day counts.) 
Lu-1380. Vojmsjöluspen, Raä 554, Sample $4 \quad 5340 \pm 70$ $\delta^{13} \mathrm{C}=-24.0 \%$

Charcoal from silty sand ca $30 \mathrm{~cm}$ below surface. Sq X112, Y318; +416.60 to 416.87 . Assoc with quartzite core and quartz scraper. Comment: $68 \%$ sample. (3 1-day counts.)

Lu-1381. Vojmsjöluspen, Raä 554, Sample $5 \quad 5550 \pm 70$ $\delta^{13} C=-24.1 \%$

Charcoal from bottom of refuse pit in humous gravel with bones. Sq X110, Y315; +416.0.

Lu-1382. Vojmsjöluspen, Raä 554, Sample $6 \quad 3410 \pm 90$ $\delta^{13} C=-23.3 \%$ o

Charcoal from layer of rust-earth, depth ca $20 \mathrm{~cm}$, in till at Brännåker 1:70. Comment: $39 \%$ sample. (3 1-day counts.)

\section{Valleberga series}

Charcoal from settlement area at Valleberga, SE Scania (Strömberg, 1978a). Coll 1973 and 1977 and subm by M Strömberg, Hist Mus, Univ Lund. For other dates from Valleberga, see R, 1974, v 16, p 324-325; 1975, v 17, p 192-193; 1976, v 18, p 313-314. Pretreated with $\mathrm{HCl}$ and $\mathrm{NaOH}$.

\section{Lu-1415. Valleberga 29:12, Sample 8:76-77 $8610 \pm 85$}

$$
\delta^{13} C=-23.6 \%
$$

Charcoal from hearth in settlement layer at Valleberga 29:12 (55 $24^{\prime} \mathrm{N}, 14^{\circ} 03^{\prime} \mathrm{E}$ ). Assoc with pottery and flints. Comment (MS): older than expected judging from archaeol finds.

\section{Lu-1416. Valleberga $33^{1}$ A, Sample 9:76-77}

$$
1060 \pm 50
$$

$$
\delta^{13} \mathrm{C}=-25.0 \% \text { o }
$$

Charcoal from pit-house at Valleberga $33^{1} \mathrm{~A}\left(55^{\circ} 25^{\prime} \mathrm{N}, 14^{\circ} 04^{\prime} \mathrm{E}\right)$. Assoc with pottery indicating Early Middle ages. Comment (MS): slightly older than expected.

\section{Lu-1417. Valleberga 50 ${ }^{4}$, Sample 10:76-77}

$$
\begin{array}{r}
3810 \pm 60 \\
\delta^{13} C=-25.2 \%
\end{array}
$$

Charcoal from hearth at base of cultural layer, Trench II, Sq $\mathbf{x}=$ $+2, \mathrm{y}=+9$ (op cit, above, p 90 and 95) at Valleberga $50^{4}\left(55^{\circ} 24^{\prime} \mathrm{N}\right.$, $14^{\circ} 04^{\prime} \mathrm{E}$ ). Assoc with flints and pottery. Comment (MS): date agrees with archaeol estimate based on assoc pottery.

\section{Ystad series}

Charcoal and bone from settlement area at Block Tankbåten in W part of Ystad town, S Scania (55 $\left.25^{\prime} \mathrm{N}, 13^{\circ} 48^{\prime} \mathrm{E}\right)$. Coll 1977 and subm by M Strömberg. Preliminary excavation rept with comment on this series pub by Strömberg (1978b). Charcoal pretreated with $\mathrm{HCl}$ and $\mathrm{NaOH}$; bone collagen extracted as described previously (R, 1976, v 18, p 290). 


\section{Lu-1435. Kv Tankbåten, Sample 11:76-77}

$$
\begin{array}{r}
1430 \pm \mathbf{5 0} \\
\delta^{13} C=-24.8 \%
\end{array}
$$

Charcoal from hearth in SW sec of Pit-house 2:77 (op cit, above, p 20, Fig 4), just below plough-disturbed soil. Assoc with animal bone and pottery.

\section{Lu-1436. Kv Tankbåten, Sample 12:76-77}

$$
\begin{array}{r}
\mathbf{1 3 1 0} \pm \mathbf{5 0} \\
\delta^{13} C=-24.6 \% \text { o }
\end{array}
$$

Charcoal from hearth at base of cultural layer in NE sec of Pit-house 2:77. Assoc with bone and pottery.

\section{Lu-1437. Kv Tankbåten, Sample 1:HT77}

$$
\begin{array}{r}
\mathbf{1 6 4 0} \pm \mathbf{5 0} \\
\delta^{13} C=-22.5 \%
\end{array}
$$

Charcoal from hearth close to and NE of Pit-house 12:77 (op cit, above, p 25, Fig 9).

Lu-1438. Kv Tankbåten, Sample 2 :HT77

$$
\begin{array}{r}
\mathbf{1 6 2 0} \pm \mathbf{5 0} \\
\delta^{1 s} C=-24.5 \% \text { o }
\end{array}
$$

Charcoal from hearth $\mathrm{E}$ of Pit-house 3:77 (op cit, above, p 21, Fig 5). Assoc with bones.

Lu-1439. Kv Tankbåten, Sample 3 :HT77

$$
\begin{array}{r}
\mathbf{1 3 1 0} \pm \mathbf{5 0} \\
\delta^{13} C=-25.0 \%
\end{array}
$$
Fig 9).

Charcoal from bottom layer in Pit-house 10:77 (op cit, above, p 25,

\section{Lu-1440. Kv Tankbåten, Sample 4:HT77}

$$
\begin{array}{r}
1210 \pm 50 \\
\delta^{13} C=-24.4 \% o
\end{array}
$$

Charcoal from oven in SW sec of Pit-house 13:77 (op cit, above, p 26-27, Figs 10-11; p 46, Fig 18). Assoc with pottery and bones.

\section{Lu-1514. Kv Tankbåten, Sample 5:HT77

$$
\delta^{13} C=-20.5 \% \text { o }
$$$$
1470 \pm 50
$$

Collagen from bone of $B o s$, id by $\mathrm{O}$ Persson, from gyttja layer in Trench III (op cit, above, p 50-52, Fig 21; p 85, Fig 38). Assoc with bone, horn, and some sherds of pottery. Comment: organic carbon content: $2.9 \%$.

General Comment (MS): dates support archaeol dating of main occupation of settlement area to Vendel period.

\section{Lödde kar series}

Wood from underwater structures assoc with Viking period harbor (Ohlsson, 1973; Lindqvist, 1976) of the mouth of Lödde R, E Scania $\left(55^{\circ} 45^{\prime} \mathrm{N}, 13^{\circ} 00^{\prime} \mathrm{E}\right)$. Coll 1975 and 1977 by T Ohlsson and P-I Lindqvist; subm by $\mathrm{T}$ Ohlsson, Hist Mus, Univ Lund. For other dates from underwater structures of similar age, see R, 1968, v 10, p 50; 1969, v 11, p 448-449; 1972, v 14, p 397-398; 1974, v 16, p 327; 1977, v 19, p 433-434. Pretreated with $\mathrm{HCl}$ and $\mathrm{NaOH}$. 
Lu-1159. Lödde kar, Log No. 1

$\mathbf{9 3 0} \pm \mathbf{5 0}$

$\delta^{13} C=-25.5 \%$ o

Unid. wood from 5 annual rings taken ca 10 rings inside bark.

Lu-1466. Lödde kar, Log No. 2

$980 \pm 50$

$\delta^{13} \mathrm{C}=-24.7 \%$

Oak wood, id by $\mathrm{T}$ Bartholin, from ca 15 of outermost annual heartwood rings. Only few sapwood rings remained uneroded.

Lu-1467. Lödde kar, Log No. 4

$\mathbf{8 9 0} \pm \mathbf{5 0}$

$\delta^{13} \mathrm{C}=-26.4 \%$

Beech wood, id by $\mathrm{T}$ Bartholin, from relatively superficial part of log with ca 35 annual rings.

\section{Löddeköpinge No. 10 series (I)}

Human bones from grave field at Löddeköpinge No. 10, Löddeköpinge parish, E Scania $\left(55^{\circ} 45^{\prime} \mathrm{N}, 13^{\circ} 00^{\prime} \mathrm{E}\right)$. Coll 1976 and subm by $\mathrm{T}$ Ohlsson. Rept from excavation of Viking age settlement at Löddeköpinge pub by submitter (Ohlsson, 1976). For other dates on material from Löddeköpinge area, see R, 1973, v 15, p 512-513; 1976, v 18, p 317-318, and Lödde kar series, above. Collagen extracted as described previously (R, 1976, p 290), but without $\mathrm{NaOH}$ treatment. Vertebrae and other bones with thin outer walls and consisting mainly of spongiose bone, were not crushed before extraction.

Lu-1398. Löddeköpinge No. 10, Structure $10 \quad 1010 \pm 45$ $\delta^{13} \mathrm{C}=-18.9 \%$

Collagen from mixture of small human bones. Comment: undersized; diluted; $82 \%$ sample. (3 1-day counts.) Organic carbon content: $6.6 \%$.

Lu-1399. Löddeköpinge No. 10, Structure $63 \quad 910 \pm 40$ $\delta^{13} \mathrm{C}=-18.3 \%$

Collagen from mixture of ill-preserved human bones. Comment: organic carbon content: $1.8 \%$. (3 I-day counts.)

Lu-1400. Löddeköpinge No. 10, Structure $76 \quad 1000 \pm 50$ $\delta^{13} C=-18.2 \%$

Collagen from 2 ankle bones, 1 vertebra, and other small human bones. Comment: organic carbon content: $7.7 \%$.

Lu-1401. Löddeköpinge No. 10, Structure $81 \quad 990 \pm \mathbf{5 0}$ $\delta^{13} \mathrm{C}=-18.4 \%$ $7.4 \%$.

Collagen from human vertebrae. Comment: organic carbon content:

Lu-1402. Löddeköpinge No. 10, Structure 94

$\mathbf{9 8 0} \pm \mathbf{5 0}$

Collagen from mixture of ill-preserved human bones. Comment: organic carbon content: $4.1 \%$. 
Lu-1403. Löddeköpinge No. 10, Structure $153 \quad 890 \pm 50$ $\delta^{13} \mathrm{C}=-18.9 \%$

Collagen from mixture of ill-preserved human bones. Comment: organic carbon content: $3.2 \%$.

Lu-1404. Löddeköpinge No. 10, Structure $155 \quad 990 \pm 50$ $\delta^{13} \mathrm{C}=-18.1 \%$

Collagen from fragments of ill-preserved human bones. Comment: organic carbon content: $3.2 \%$.

Lu-1405. Löddeköpinge No. 10, Structure 164 $990 \pm 50$ $\delta^{13} \mathrm{C}=-18.5 \%$

Collagen from mixture of ill-preserved small human bones. Comment: organic carbon content: $3.4 \%$.

Lu-1406. Löddeköpinge No. 10, Structure $179 \quad 1010 \pm 50$ $\delta^{13} \mathrm{C}=-18.6 \%$

Collagen from 2 heel bones, 2 ankle bones, 2 kneecaps, and some other small human bones. Comment: organic carbon content: $6.4 \%$.

Lu-1407. Löddeköpinge No. 10, Structure $211 \quad 1000 \pm 50$ $\delta^{13} \mathrm{C}=-18.8 \%$

Collagen from 1 heel bone, 1 ankle bone, 2 kneecaps and some other small human bones. Comment: organic carbon content: $8.2 \%$.

Lu-1408. Löddeköpinge No. 10, Structure $216 \quad 1050 \pm 50$ $\delta^{13} \mathrm{C}=-18.9 \%$

Collagen from 2 heel bones, 2 ankle bones, and some other small human bones. Comment: organic carbon content: $6.8 \%$.

Lu-1409. Löddekopinge No. 10, Structure $237 \quad 950 \pm 50$ $\delta^{13} \mathrm{C}=-18.5 \%$

Collagen from tubular human bones. Comment: organic carbon content: $7.8 \%$.

Lu-1410. Löddeköpinge No. 10, Structure $248 \quad \mathbf{1 1 3 0} \pm \mathbf{5 0}$

Collagen from 1 heel bone, 1 ankle bone, and some human vertebrae. Comment: organic carbon content: $6.2 \%$.

Lu-1411. Löddeköpinge No. 10, Structure $251 \quad 990 \pm 50$ $\delta^{13} \mathrm{C}=-18.4 \%$

Collagen from ill-preserved human femur. Comment: organic carbon content: $3.3 \%$.

Lu-1412. Löddeköpinge No. 10, Structure $272 \quad 910 \pm 50$ $\delta^{13} \mathrm{C}=-19.0 \%$

Collagen from fragments of human vertebrae and heel bones. Comment: organic carbon content: $5.7 \%$. 
Lu-1413. Löddeköpinge No. 10, Structure 274 $1030 \pm 50$ $\delta^{13} \mathrm{C}=-18.9 \%$

Collagen from mixture of ill-preserved small human bones: Comment: organic carbon content: $3.7 \%$.

\section{Lu-1414. Löddeköpinge No. 10, Structure $311 \quad 970 \pm 50$}

$\delta^{13} \mathrm{C}=-18.6 \%$

Collagen from fragments of ill-preserved vertebrae, rib bones, and other small human bones. Comment: organic carbon content: $3.0 \%$.

General Comment: all dates are older than expected judging from coins found in some graves. Bones from those graves will be dated to give information about age discrepancies, which are too large to be explained by ${ }^{14} \mathrm{C}$ variations and apparent age of bone collagen in living adult humans due to the slow turnover in bone material ( $R, 1972$, v 14, p 112). Discrepancies may possibly be explained by influence from frequent use of marine food, which is deficient in ${ }^{14} \mathrm{C}$ compared to food from terrestrial environments when it is normalized to the same ${ }^{13} \mathrm{C} /{ }^{12} \mathrm{C}$ ratio (R, 1972, v 14, p 112-113).

\section{Ageröd series (II)}

Charcoal and bone from Mesolithic settlement area at raised bog Ageröds mosse, Munkarp parish, Scania $\left(55^{\circ} 56.5^{\prime}\right.$ N. $13^{\circ} 25^{\prime}$ E). Coll 1974 and 1977 and subm by L Larsson, Hist Mus, Univ Lund. Results of archaeol study pub by submitter (Larsson, 1978). Dated as complement to Ageröd series (R, 1976, v 18, p 304-308). Charcoal id by $\mathrm{T}$ Bartholin; bone id by O Persson. No pretreatment of charcoal samples; undersized; diluted. Collagen extracted from bone sample as described previously (R, 1976, v 18, p 290).

\section{Lu-1499. Ageröd I:HC, Sample 15}

$$
\begin{array}{r}
\mathbf{7 8 2 0} \pm \mathbf{9 0} \\
\delta^{13} \mathrm{C}=-24.9 \%
\end{array}
$$

Charcoal (Ulmus, Corylus, and Quercus) from bottom layer. Comment: $84 \%$ sample.

\section{Lu-1500. Ageröd I:HC, Sample 16}

$$
\begin{array}{r}
\mathbf{7 2 0 0} \pm \mathbf{9 0} \\
\delta^{13} \mathrm{C}=-24.9 \%
\end{array}
$$

Charcoal (Corylus, Alnus, Quercus, and Salix or Populus) from upper peat. Comment: $75 \%$ sample.

\section{Lu-1502. Ageröd V, Sample 4}

$$
\begin{array}{r}
\mathbf{6 7 1 0} \pm \mathbf{7 0} \\
\delta^{13} C=-21.4 \%
\end{array}
$$

Collagen from fragment of scapula of red deer from refuse layer, Sq C17. Comment: organic carbon content: $5.5 \%$.

\section{Lu-1501. Segebro, Sample 6}

$$
7140 \pm 75
$$

$$
\delta^{13} C=-24.0 \%
$$

Charcoal (Corylus, Ulmus, and Tilia) from hearth on Mesolithic settlement Segebro in delta of Sege R, SW Scania ( $55^{\circ} 37^{\prime} 25^{\prime \prime}$ N, $13^{\circ} 03^{\prime}$ 
35" E). Coll 1976 and subm by L Larsson. Dated as complement to Segebro series (R, 1976, v 18, p 308-309).

\section{Saxtorp series}

Charcoal from Mesolithic pit-house and refuse pit (Larsson, 1975)

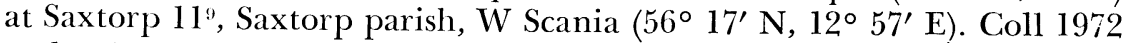
and subm by L Larsson. Assoc finds of arrowheads and other worked flints indicate Kongemose culture. Pretreated with $\mathrm{HCl}$ and $\mathrm{NaOH}$.

Lu-1524. Saxtorp $11^{9}$, Sample 1

$6970 \pm 70$

$\delta^{13} C=-23.1 \%$

Charcoal (Pinus) from pit-house bottom. Assoc with oblique arrowheads and flint waste.

Lu-1525. Saxtorp $11^{9}$, Sample 2

$$
\begin{array}{r}
4860 \pm 65 \\
\delta^{13} C=-24.2 \% \text { 。 }
\end{array}
$$

Charcoal (Quercus) from small refuse pit. Assoc with oblique arrowheads, transverse arrowhead, and flint waste.

\section{Lu-1464. Källby}

Collagen from horse tibia found in secondary position assoc with human bones during excavation for artificial dam at Källby, Lund, SW Scania $\left(55^{\circ} 41^{\prime} \mathrm{N}, 13^{\circ} 10^{\prime} \mathrm{E}\right)$. Coll 1971 by O Persson, who id the bones; subm by J Callmer, Hist Mus, Univ Lund.

\section{REFERENCES}

Berge, J, Bostwick, L G, Krzywinski, K, Myhre, B, Stabell, B, and Ågotnes, A, 1978, Ilandføring av olje på Sotra. De arkeologiske undersøkelser 1977: Vindenes, Hist Mus, Univ Bergen, Rept, 292 p.

Bergersen, O F and Garnes, K, 197 i, Evidence of sub-till sediments from a Weichselian interstadial in the Gudbrandsdalen Valley, Central East Norway: Norsk Geog Tidsskr, v 25, p 99-108.

Berglund, B E, 1966, Late-Quaternary vegetation in eastern Blekinge, southeastern Sweden. II. Post-Glacial time: Op Bot a Soc Lundensi, v 12, no. 2, 190 p.

1978, Landskapsförändringar i Östblekinge. 2. Vieskär och öppenskär i Torhamns skärgård: Blekinges Natur 1978 (Karlskrona), p 15-36.

Berglund, B E, Håkansson, Sören, and Lagerlund, Erik, 1976, Radiocarbon-dated mammoth (Mammuthus primigenius, Blumenbach) finds in South Sweden: Boreas (Olso), v 5, p 177-191.

Garnes, Kari, 1978, Zur Stratigraphie der Weichseleiszeit im zentralen Südnorwegen, in: Schneiderbauer, $\mathbf{H}$ and Nagl, $\mathbf{H}$ (eds), 1978, Beiträge zur Quartär - und Landschaftsforschung. Festschrift zum 60. Geburtstag von Julius Fink, Verlag Ferdinand Hirt, Wien, p 195-220.

Girard, Michel, 1970, Analyse pollinique de l'Interglaciaire Riss-Würm de Sous-Terre à Genève (Suisse): Soc Phys et Hist Nat, Genc̀ve, Compte rendu, ns, v 5, Fasc 1, p $70-74$.

Håkansson, Sören, 1968, University of Lund radiocarbon dates I: Radiocarbon, v 10 , p $36-54$.

_ _ 1969, University of Lund radiocarbon dates II: Radiocarbon, v 11, p 430-450. 1972, University of Lund radiocarbon dates V: Radiocarbon, v 14, p 380-400. 513. 1973, University of Lund radiocarbon dates VI: Radiocarbon, v 15, p 493513.

330 . 1974, University of Lund radiocarbon dates VII: Radiocarbon, v 16, p 307p 174-195. 
Håkansson, Sören, 1975b, Radiocarbon dating of shell samples from Western Sweden, Appendix, in Hillefors, Ake, 1975, Contributions to the knowledge of the chronology of the deglaciation of Western Sweden with special reference to the Gothenburg moraine: Svensk Geog Årsb, Arg 51, p 78-80.

320. 1976, University of Lund radiocarbon dates IX: Radiocarbon, $v 18, \mathrm{p} 290$

— 1977, University of Lund radiocarbon dates X: Radiocarbon, v 19, p 424-441. 435.

Harkness, D D and Walton, A, 1972, Glasgow University radiocarbon measurements IV: Radiocarbon, v 14, p 111-113.

Hillefors, Ake, 1975, Contributions to the knowledge of the chronology of the deglaciation of Western Sweden with special reference to the Gothenburg moraine: Svensk Geog Årsb, Årg 51, p 70-81.

Jayet, Adrien and Amberger, Gad, 1969, L'Interglaciaire Riss-Würm de Sous-Terre à Genève: Eclogae geol Helvetiae (Basel), v 62, no. 2, p 629-636.

Klimaszewski, M, 1960, Studia geomorfologiczne w zachodniej czéści Spitsbergenu miçdzy Kongs-Fjordem a Eidem Bukta: Zeszyty Naukowe Uniw Jagiellońskiego, 32, Prace Geog, Ser Nowa 1, Kraków, 166 p.

Krzywinski, Knut and Stabell, Bjørg, 1978, Senglasiale undersøkelser på Sotra: Arkeo, arkeol medd fra Hist Mus, Univ Bergen, no. 1, 1978, p 27-31.

Larsson, Lars, 1975, A contribution to the knowledge of Mesolithic huts in soushern Scandinavia: Lunds Univ Hist Mus Medd 1973-1974, p 5-28.

1978, Ageröd I:B - Ageröd I:D. A study of Early Atlantic settlement in Scania: Acta Archaeol Lundensia, ser in $4^{\circ}$, No. 12, 258 p.

Lindqvist, P-I, 1976, Marinarkeologi i Öresund: Ale, Hist Tidsskr för Skåneland, 1976, no. 1 , p 17-29.

Mikaclsson, Jan, 1978, Strandvallskomplexet vid Olsäng: Blekinges Natur 1978 (Karlskrona), p $37-52$.

Mikaelsson, Jan, and Liljegren, Ronnic, 1978, öja-Herrestads mosse: Våra Härader 1978, Ljunits och Herrestads Hembygdsförening, no. 11, p 16-22.

Ohlsson, Tom, 1973, Vikingatid och medeltid i Löddeköpinge: Ale, Hist, Tidsskr för Skåneland, 1973, no. 1, p 27-42.

1976, The Löddeköpinge investigation I. The settlement at Vikhögsvägen: Lunds Univ Hist Mus Medd 1975-1976, ns, v 1, p 59-161.

Strömberg, Märta, 1978a, Three Neolithic sites. A local seriation?: Lunds Univ Hist Mus Medd 1977-78, ns, v 2, p 68-97.

1978b, En kustby i Ystad före stadens tillkomst: Ystads Fornminnesförenings årsb, v 23, p 11-101. 